\title{
An FEM-Based State Estimation Approach to Nonlinear Hybrid Positioning Systems
}

\author{
Yu-Xin Zhao, ${ }^{1,2}$ Li-Juan Chen, ${ }^{1}$ and Yan $\mathrm{Ma}^{1}$ \\ ${ }^{1}$ College of Automation, Harbin Engineering University, Harbin 150001, China \\ ${ }^{2}$ Center for Transport Studies, Imperial College, London SW7 2AZ, UK
}

Correspondence should be addressed to Yu-Xin Zhao; zhaoyuxin@hrbeu.edu.cn

Received 2 April 2013; Accepted 25 May 2013

Academic Editor: Xiaojie Su

Copyright (c) $2013 \mathrm{Yu}$-Xin Zhao et al. This is an open access article distributed under the Creative Commons Attribution License, which permits unrestricted use, distribution, and reproduction in any medium, provided the original work is properly cited.

\begin{abstract}
For hybrid positioning systems (HPSs), the estimator design is a crucial and important problem. In this paper, a finite-elementmethod- (FEM-) based state estimation approach is proposed to HPS. As the weak solution of hybrid stochastic differential model is denoted by the Kolmogorov's forward equation, this paper constructs its interpolating point through the classical fourth-order Runge-Kutta method. Then, it approaches the solution with biquadratic interpolation function to obtain a prior probability density function of the state. A posterior probability density function is gained through Bayesian formula finally. In theory, the proposed scheme has more advantages in the performance of complexity and convergence for low-dimensional systems. By taking an illustrative example, numerical experiment results show that the new state estimator is feasible and has good performance than PF and UKF
\end{abstract}

\section{Introduction}

In recent years, a new concept called hybrid positioning systems (HPSs) is more and more popular in navigation and location-based services research community [1]. HPSs are systems for finding the location of a mobile device by using several different positioning technologies in indoor environments. Mostly, GPS is one major component of such systems, aided by others such as tower signals, wireless internet signals, Indoor Messaging System (IMES), bluetooth sensors, or other local positioning systems $[1,2]$. So far many different hybrid positioning systems are being explored and gradually applied in services from Google Maps for Mobile, Combain Mobile, SkyHook, openBmap, Navizon, PlaceEngine, Xtify, and so forth.

Obviously, HPS will be well developed in urban locationbased service areas because of the huge commercial market. So studies are rapidly increasing and more practically viable than ever $[1,3-6]$. But in these literatures, researchers paid more attention to the architecture of HPS, or how to choose and integrate different sensors or signals reliably $[1,5,6]$. Actually, for getting reasonable accuracy and precision, position estimator is one of the key factors in the hybrid positioning systems, which will directly affect the quality of system performance. As we all know, for an HPS use in real environments, there is hardly enough measuring information for a unique position because of the reflection conditions of the environment, which will influence the WLAN or the other radio signals. On the other hand, the measurement functions are nonlinear functions of the motion state, so we have to think about the estimation accuracy the variations because the sensors' signals may significantly change depending on the motion of vehicle (or person) and slight differences of the positions at which the signals are measured [7]. For example, due to the influence of the transmission distance, WLAN or other mobile phone signals with the nonlinearity can have a significant effect on the estimation errors than GPS measurements.

Since state estimation of a stochastic dynamic system from noisy observations is a crucial and important problem in hybrid positioning applications, the motivation for this paper is what we can do with the performance of position estimator. In [8] three Kalman Filter Extensions in hybrid navigation are evaluated, respectively. It is not difficult to find that the estimates tend to become biased and inconsistent when the linearization errors are coming large. Subsequently, 
a robust Extended Kalman Filtering modeled in [9] shows that it outperforms EKF and EKF2 in cases where there is blunder measurement or considerable linearization errors present only in simulation cases. Although EKF is popular in many navigation applications [10-15], it must satisfy three assumptions for using EKF and its variants. (1) The deviations of the reference state trajectory should be small. (2) The mathematical description for state and observation of the dynamics system should be accurate. (3) The conditional density function of the state should be Gaussian. Otherwise, the performance of EKF will become unstable and poorly. Therefore, this is not a recommended approach for HPS estimating problems.

Particle Filter (PF) is another popular estimating algorithm in HPS $[1,6]$, which is also known as a sophisticated technique based on Sequential Monte Carlo (SMC) method, started by Gordon et al. in 1993 [16]. Roughly speaking, the power of $\mathrm{PF}$ is that it can approximate the posterior probability density of a complex system by using a kind of stochastic sampling particles with different weights and update the posterior by involving the new observations according to the Bayesian principle. In the past twenty years, a lot of work has been done to develop the performance of particle filters. Especially like Auxiliary Particle Filter [17], Unscented Particle Filter [18], and Gaussian Particle Filtering [19] et al. improve the importance sampling effectively. In [20], a so-called Rao-Blackwellised Particle Filtering can exploit the structure of the dynamic Bayesian networks to increase the efficiency of particle filtering. For preventing the sample impoverishment problem, some heuristic algorithms like genetic algorithm (GA) are incorporated into a particle filter to overcome this drawback of the filter innovatively [21]. With the development of computing technology, PF is now in a golden age as it could deal with nonlinear, non-Gaussian, non-steady-state recursive estimation problem. However, in fact, it still has some disadvantages in practice, such as the fast growing computational complexity and the sample impoverishment.

Theoretically speaking, a typical hybrid positioning system should be described by a partial differential equation (Kolmogorov's forward equation) and a difference equation separately, while the former reflects how the conditional density of a dynamic system evolves, and the latter means how it is works by the new measuring information [22]. For solving the problem, finite element method (FEM), a classic procedure for approximating solutions of partial differential equations (PDEs), is introduced in this paper. FEM assumes that the exact solution to a PDE could be expanded with a sum of local basis functions. Inspired by [23-26], an approximate solution is found by combining interpolation points and shape function. Essentially, FEM has more advantages than $\mathrm{PF}$ or other classic filters like EKF and UKF. It outperforms others in case of model mismatches, large state variations, and arbitrary initial conditions.

This paper is organized as follows. The next section consists of the abstractive description of the hybrid positioning problem in a Bayesian framework. The measurement model and the state model are described as continuous nonlinear dynamics and discrete nonlinear observations separately.
Section 3 gives the procedure of FEM and shows how make it works to solve estimating problems. In Sections 4 and 5 , we analyze the algorithm performance of convergence and computational complexity mathematically. Then, an illustrative example and its simulation results are given in Section 6. Finally, we conclude this paper in Section 7.

\section{Problem Description and Preliminaries}

Actually, most hybrid positioning systems that we have in real applications may be reasonably described in continuous dynamics and discrete measurements. So an abstractive model denoted by a partial differential equation and a difference equation separately as

$$
\begin{gathered}
d x_{t}=f\left(x_{t}, t\right) d t+g\left(x_{t}, t\right) d \beta_{t}, \quad t \geq t_{0}, \\
z_{k}=h\left(x_{t_{k}}, t_{k}\right)+e_{k}
\end{gathered}
$$

is considered, where $x_{t} \in \mathbb{R}^{n}$ is a state vector and $z_{k} \in \mathbb{R}^{m}$ is an output vector. $f\left(x_{t}, t\right): \mathbb{R}^{n} \times \mathbb{R} \rightarrow \mathbb{R}^{n}$ and $h\left(x_{t_{k}}, t_{k}\right): \mathbb{R}^{n} \times$ $\mathbb{R} \rightarrow \mathbb{R}^{m}$ denote system and measurement equations, while $g\left(x_{t}, t\right): \mathbb{R}^{n} \times \mathbb{R} \rightarrow \mathbb{R}^{n \times d}$ is diffusion coefficient. Here $\beta_{t}$ is $d$-dimensional Brownian motion vector with the covariance matrix $E\left[d \beta_{t} d \beta_{t}^{T}\right]=Q(t) d t$, and $e_{k}$ is an $m$-dimensional white Gaussian variable independent of $d \beta_{t}$ with covariance matrix $R(k), k \geq 1$, where $E\left[e_{k}^{T} e_{k}\right]=R(k), R(k) \in \mathbb{R}^{m \times m}$.

Let $Z_{k}=\left\{z_{k}, t_{k} \leq t\right\}$ denote the observations set accumulated up to $k$, so the problem is evolved into how to seek for the conditional density $p\left(x, t \mid z_{t}\right)$. Because the statistical information of the system is contained in the measurements $z_{k}$ and the initial condition $p\left(x_{0}, t_{0} \mid z_{0}\right)$, the conditional mean and variance can be computed from $p\left(x, t \mid z_{t}\right)$, which generally depend on all of the higher order moments for any nonlinear system. Theoretically, with the observations between time $t_{k}$ and $t_{k+1}$, the conditional density $p \triangleq p\left(x, t \mid z_{t}\right)$ diffuses according to Kolmogorov's forward equation

$$
\frac{\partial p}{\partial t}=\frac{1}{2} \cdot \frac{\partial^{2}\left(\sigma^{2}(y, t) p\right)}{\partial y^{2}}-\frac{\partial(\mu(y, t) p)}{\partial y} .
$$

Usually, a weak solution of stochastic differential equation could be decided by transferring function, so the conditional probability density $p\left(x, t \mid z_{t}\right)$ of system will satisfy Kolmogorov's forward equation

$$
\begin{aligned}
L(p)= & \dot{p} \\
= & \frac{1}{2} \sum_{r, s=1}^{n} \frac{\partial^{2}\left(g(x, t) Q(t) g^{T}(x, t) \cdot p\right)}{\partial x_{r} \partial x_{s}} \\
& -\sum_{r=1}^{n} \frac{\partial(f(x, t) \cdot p)}{\partial x_{r}},
\end{aligned}
$$

where $p \triangleq p\left(x, t \mid z_{t}\right)$ is called the weak solution of the stochastic differential model. Consider

$$
p\left(x_{0}, t_{0} \mid z_{0}\right)=p_{0} .
$$


Thus, Kolmogorov's forward equation (2) and its initial boundary conditions (3) have been differential equations boundary value problems, where $\Omega$ is smooth boundaries area in $\mathbb{R}^{n}$, and $P: \Omega \rightarrow \mathbb{R}$ is solution of boundary value problem.

\section{An Estimator Design Based on Finite Element Method}

In fact, with the recursive Bayesian estimation framework, Particle Filter is a method that piecewise approximates probability density function of a state in function space, while finite element method (FEM) approximates for state distribution function in the state space. When we approximate the solution of Kolmogorov's forward equation by FEM, it could be conducted into a problem to solve the ordinary differential equations with coefficients in piecewise function. In this section, firstly, we use FEM to simplify formula (2) from a function of space and time to a function of time only, and a linear ordinary differential equation is obtained, which is easy to solve numerically. Certainly, FEM is a powerful tool to solve Partial Differential Equations (PDEs) problem, which is discussed in most textbooks; therefore, an estimator design flowchart based on FEM is shown in Figure 1, reference to the literature [24-26].

For any state vector $x\left(x_{i} \in R\right)$, the probability density function of state can be calculated as

$$
p\left(x, \frac{t}{z_{k}}\right): R^{n} \times\left[t_{0}, t_{e}\right) \longrightarrow R^{2},
$$

with a fixed time $t$, where $p(x, t)$ is a member of the Hilbert space that is consisted with $f: R^{n} \rightarrow R^{2}$, so it holds that

$$
p\left(x, \frac{t}{z_{k}}\right) \triangleq p(x, t) .
$$

3.1. Biquadratic Interpolation Method. In a two-dimensional space, according to the mean value $E_{k}$ and variance $\sigma_{k}$ with the previous moment $t_{k}$, given an interval $\left\{\left(x_{1}, x_{2}\right) \mid a \leq x \leq\right.$ $b, c \leq y \leq d\}$, we can get a rectangular area with $\left\{\left(x_{1}, x_{2}\right) \mid\right.$ $\left.a_{i-1}<x_{1}<a_{i}, b_{j-1}<x_{2}<b_{j}, i=1, \ldots, n_{1}, j=1, \ldots, n_{2}\right\}$ as shown in Figure 2.

In Figure 2(a), for any quadrilateral unit, it can be expressed as $\left(\xi_{i}, \zeta_{i}\right), i=1, \ldots, 4$, respectively, whose function values are given as $p_{1}, p_{2}, p_{3}$, and $p_{4}$. Assuming that only four corner nodes are established at the beginning, so the corresponding shape function by Lagrange polynomial can be obtained as

$$
\widetilde{N}_{i}=\frac{\left(1+\xi_{i} \cdot x_{1}\right) \cdot\left(1+\zeta_{i} \cdot x_{2}\right)}{4}, \quad i=1, \ldots, 4 .
$$

The shape function of each node should satisfy the conditions (8)

$$
N_{i}(j)= \begin{cases}1 & i=j, \\ 0 & i \neq j\end{cases}
$$

where $j$ represents the number of nodes.
If the edge node is added, the corresponding interpolation function can be directly represented as the Lagrange polynomial product of a quadratic in $x_{1}$ ( or $x_{2}$ ) direction and a first degree polynomials in $x_{2}$ (or $x_{1}$ ) direction. Thus, the shape function of the fifth adding node can be described as

$$
N_{5}=\frac{\left(1-x_{2}^{2}\right) \cdot\left(1-x_{1}\right)}{2} \text {. }
$$

For the previous five nodes, obviously, $N_{5}(j)$ satisfies the requirements of the shape function, but node 1 and node 2 cannot meet the requirements as formula (8). In order to make them work properly, $\widetilde{N_{1}}, \widetilde{N_{2}}$ should be modified as follows:

$$
N_{1}=\widetilde{N_{1}}-\frac{N_{5}}{2}, \quad N_{2}=\widetilde{N_{2}}-\frac{N_{5}}{2}
$$

where the coefficient $1 / 2$ means the value at the node 5 with $\widetilde{N_{1}}, \widetilde{N_{2}}$.

Similarly, we can add the other new nodes, so all of nodes' interpolation shaper functions can be obtained as follows:

$$
\begin{array}{cc}
N_{1}=\widetilde{N_{1}}-\frac{N_{5}}{2}-\frac{N_{8}}{2}, & N_{2}=\widetilde{N_{2}}-\frac{N_{5}}{2}-\frac{N_{6}}{2}, \\
N_{3}=\widetilde{N_{3}}-\frac{N_{6}}{2}-\frac{N_{7}}{2}, & N_{4}=\widetilde{N_{4}}-\frac{N_{7}}{2}-\frac{N_{8}}{2}, \\
N_{5}=\frac{\left(1-x_{2}^{2}\right) \cdot\left(1-x_{1}\right)}{2}, & N_{6}=\frac{\left(1-x_{1}^{2}\right) \cdot\left(1-x_{2}\right)}{2}, \\
N_{7}=\frac{\left(1-x_{2}^{2}\right) \cdot\left(1+x_{1}\right)}{2}, & N_{8}=\frac{\left(1-x_{1}^{2}\right) \cdot\left(1+x_{2}\right)}{2} .
\end{array}
$$

In addition, $N_{1}, N_{2}, N_{3}, N_{4}$ can be simplified as

$$
\begin{aligned}
& N_{1}=\frac{\left(1+x_{2}\right) \cdot\left(1-x_{1}\right) \cdot\left(x_{2}-x_{1}-1\right)}{4}, \\
& N_{2}=\frac{\left(x_{2}-1\right) \cdot\left(1-x_{1}\right) \cdot\left(x_{2}+x_{1}+1\right)}{4}, \\
& N_{3}=\frac{\left(1-x_{2}\right) \cdot\left(1+x_{1}\right) \cdot\left(x_{1}-x_{2}-1\right)}{4}, \\
& N_{4}=\frac{\left(x_{2}+1\right) \cdot\left(1+x_{1}\right) \cdot\left(x_{2}+x_{1}-1\right)}{4} .
\end{aligned}
$$

Furthermore, the previous shape functions can be expanded as

$$
\begin{aligned}
N_{i}= & a_{i 1}+a_{i 2} \cdot x_{1}+a_{i 3} \cdot x_{2}+a_{i 4} \cdot x_{1}^{2}+a_{i 5} \cdot x_{1} \cdot x_{2} \\
& +a_{i 6} \cdot x_{2}^{2}+a_{i 7} \cdot x_{1}^{2} \cdot x_{2}+a_{i 8} \cdot x_{1} \cdot x_{2}^{2}, \quad i=1, \ldots, 8
\end{aligned}
$$

So the coefficients can be obtained by solving these shape function equations.

In Figure 2(b), the subunit coordinates of any quadrilateral are expressed by $\left(x_{1}, y_{1}\right), \ldots,\left(x_{8}, y_{8}\right)$, respectively, and 


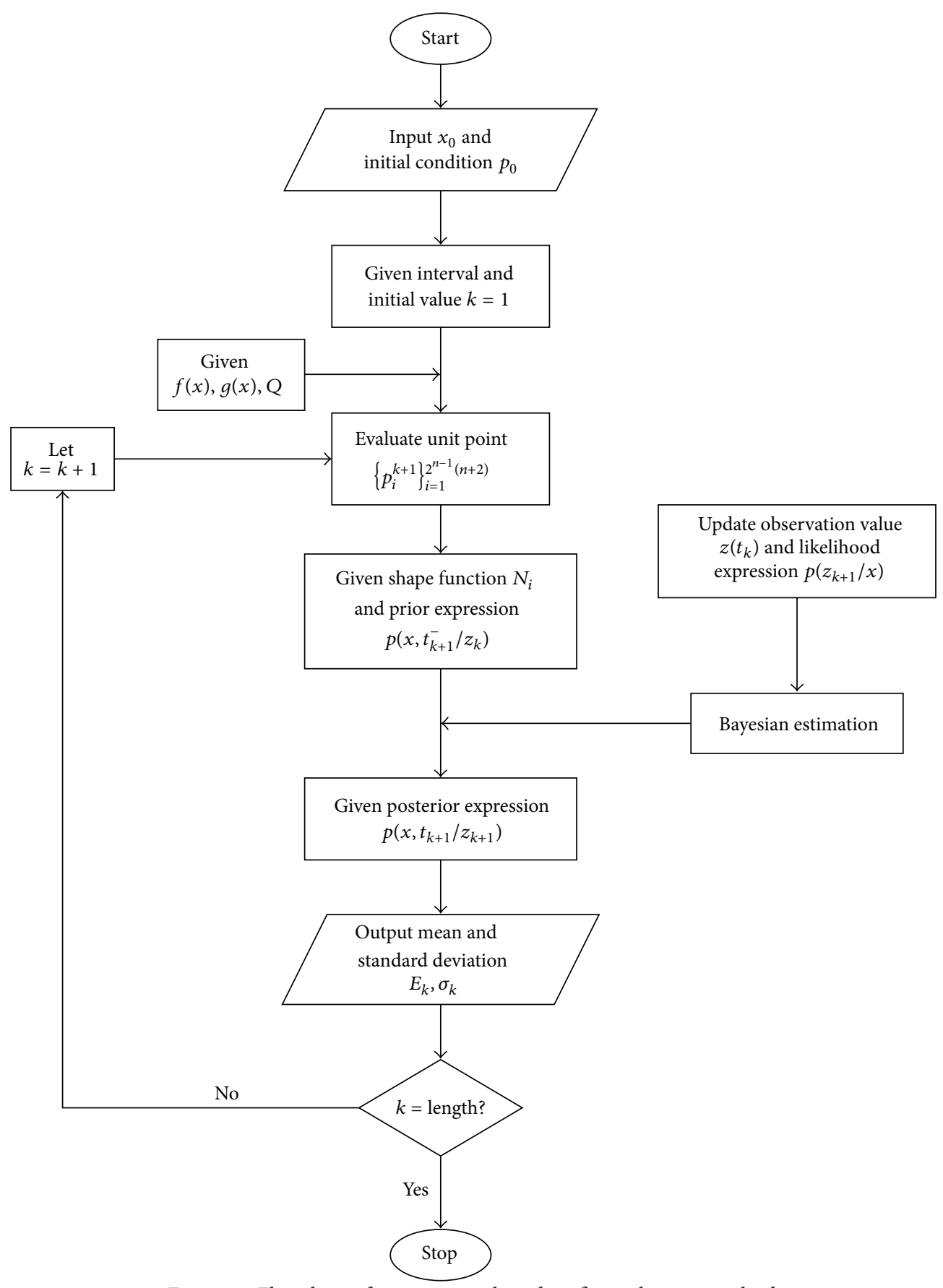

FIGURE 1: Flowchart of an estimator based on finite element method.

their probability density values are $p_{1}, p_{2}, \ldots, p_{8}$, so we can get the values of any subunit $p, x$, and $y$ as follows:

$$
p=\sum_{i=1}^{8} N_{i} \cdot p_{i}, \quad x=\sum_{i=1}^{8} N_{i} \cdot x_{i}, \quad y=\sum_{i=1}^{8} N_{i} \cdot y_{i}
$$

Contrast with the traditional generalized coordinate finite element models, the biquadratic interpolation algorithm is more simple and effective. None else by seeking for the value of the interpolation points, it can build arbitrary quadrilateral element interpolation function with the coordinate system of rectangular unit type. It not only avoids the matrix inverse calculation, the integral of the cell matrix can also be used in a standardized manner in the specification domain in a Bayesian formula.

3.2. Construction of Interpolation Points. Suppose that a Kolmogorov's forward equation is defined as follows:

$$
\begin{aligned}
L(p)= & \frac{\partial p}{\partial t} \\
= & \frac{1}{2} \sum_{r, s=1}^{n} \frac{\partial^{2}\left(g(x, t) Q(t) g^{T}(x, t) \cdot p\right)}{\partial x_{r} \partial x_{s}} \\
& -\sum_{r=1}^{n} \frac{\partial(f(x, t) \cdot p)}{\partial x_{r}} .
\end{aligned}
$$




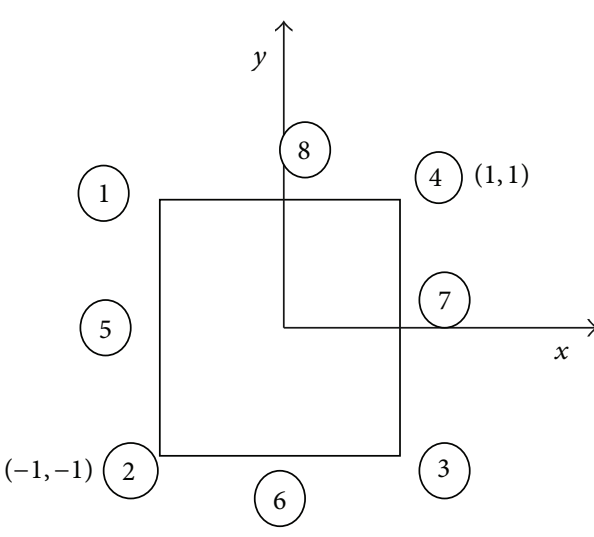

(a)

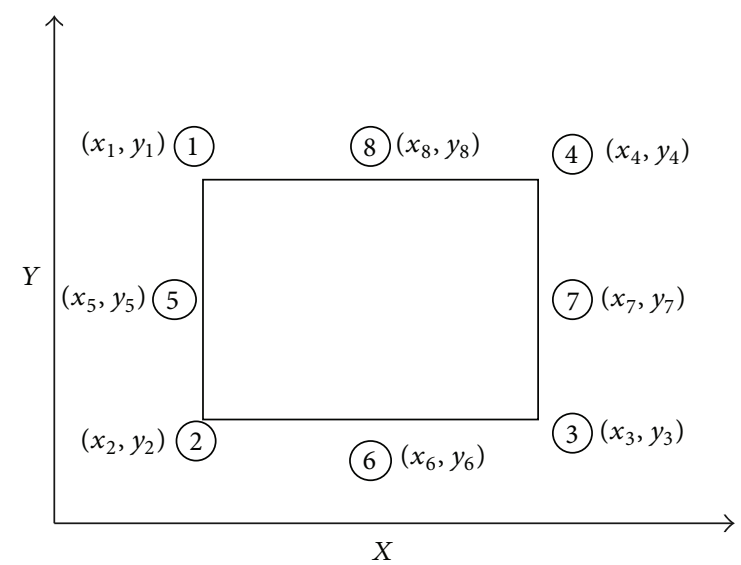

(b)

Figure 2: (a) A parent element of FEM. (b) Subunits of FEM.

Let $g(x, t) \triangleq g, Q(t) \triangleq Q, f(x, t) \triangleq f$, and due to

$$
\begin{aligned}
& \frac{\partial(f \cdot p)}{\partial x_{r}}=\frac{\partial(f)}{\partial x_{r}} \cdot p+f \cdot \frac{\partial(p)}{\partial x_{r}}, \\
& \frac{\partial\left(g Q g^{T} \cdot p\right)}{\partial x_{s}}=\frac{\partial\left(g Q g^{T}\right)}{\partial x_{s}} \cdot p \\
& +g Q g^{T} \cdot \frac{\partial(p)}{\partial x_{s}}, \\
& \frac{\partial^{2}\left(g Q g^{T} \cdot p\right)}{\partial x_{r} \partial x_{s}}=\frac{\partial^{2}\left(g Q g^{T}\right)}{\partial x_{r} \partial x_{s}} \cdot p+\frac{\partial\left(g Q g^{T}\right)}{\partial x_{r}} \cdot \frac{\partial(p)}{\partial x_{s}} \\
& +g Q g^{T} \cdot \frac{\partial^{2}(p)}{\partial x_{r} \partial x_{s}}+\frac{\partial\left(g Q g^{T}\right)}{\partial x_{s}} \cdot \frac{\partial(p)}{\partial x_{r}},
\end{aligned}
$$

we can get

$$
\begin{aligned}
& L(p(x))=\frac{\partial p}{\partial t} \\
&=\frac{1}{2} \sum_{r, s=1}^{n}\left[\frac{\partial^{2}\left(g Q g^{T}\right)}{\partial x_{r} \partial x_{s}} \cdot p+2 \cdot \frac{\partial\left(g Q g^{T}\right)}{\partial x_{r}} \cdot \frac{\partial(p)}{\partial x_{s}}\right. \\
&\left.\quad+g Q g^{T} \cdot \frac{\partial^{2}(p)}{\partial x_{r} \partial x_{s}}\right] \\
&-\sum_{r=1}^{n}\left[\frac{\partial(f)}{\partial x_{r}} \cdot p+f \cdot \frac{\partial(p)}{\partial x_{r}}\right] .
\end{aligned}
$$

Thus,

$$
\begin{gathered}
\frac{1}{2} \sum_{r, s=1}^{n} \frac{\partial^{2}\left(g Q g^{T}\right)}{\partial x_{r} \partial x_{s}}-\sum_{r=1}^{n} \frac{\partial(f)}{\partial x_{r}} \triangleq \Delta_{1}, \\
\sum_{r=1}^{n} \frac{\partial\left(g Q g^{T}\right)}{\partial x_{r}}-f \triangleq \Delta_{2}, \quad g Q g^{T} \triangleq \Delta_{3} .
\end{gathered}
$$

If $g, Q, f$ are independent of time $t$, so $\Delta_{1}, \Delta_{2}, \Delta_{3}$ are independent of $t$ as well. When $n=2$, assume that a two-dimensional vector is defined as $x=\left(x_{1}, x_{2}\right)$, and the following equation can hold:

$$
\begin{array}{rl}
\int_{t_{k}}^{t_{k+1}} & L(p(x)) \\
& =\int_{t_{k}}^{t_{k+1}} \frac{\partial p}{\partial t} d t \\
& =\int_{t_{k}}^{t_{k+1}}\left(\Delta_{1} p+\Delta_{2} \sum_{i=1}^{2} \frac{\partial p}{\partial x_{i}}+\Delta_{3} \sum_{i, j=1}^{2} \frac{\partial^{2}(p)}{\partial x_{i} \partial x_{j}}\right) d t,
\end{array}
$$

where $\left(t_{k+1}-t_{k}\right) \triangleq \Delta t, \Delta_{1} p+\Delta_{2} \sum_{i=1}^{2}\left(\partial p / \partial x_{i}\right)+\Delta_{3} \sum_{i, j=1}^{2}$ $\left(\partial^{2}(p) / \partial x_{i} \partial x_{j}\right) \triangleq F(p, t)$.

By using the Runge-Kutta method, the probability density can be calculated as

$$
p^{t_{k+1}}(x)=p^{t_{k}}(x)+\frac{\Delta t}{6} \cdot\left(K_{1}+2 K_{2}+2 K_{3}+K_{4}\right),
$$

where

$$
\begin{gathered}
K_{1}=F\left(p, t_{k}\right), \\
K_{2}=F\left(p, t_{k}\right)+\frac{\Delta t}{2} \cdot K_{1}, \\
K_{3}=F\left(p, t_{k}\right)+\frac{\Delta t}{2} \cdot K_{2}, \\
K_{4}=F\left(p, t_{k}\right)+\Delta t \cdot K_{3} .
\end{gathered}
$$


Thus, by substituting $K_{i}$ into (20), we can get the probability density of unit point $p_{i i}{ }^{t_{k+1}}\left(X_{i j}\right)$ at time $t_{k+1}$ in any rectangular area.

3.3. Bayesian Estimation. Suppose that $p^{t_{k+1}} \triangleq p_{i}^{k+1}$, so the probability density of subunit area can be formulated as

$$
p_{i j}=\sum_{k=1}^{8} N_{k} \cdot p_{i j}^{k}
$$

where $p_{i j}^{k}$ represents the probability density value of node $k$ at time $t_{k+1}$ in a rectangular area.

So the prior probability density expression $p(x, t)$ can be obtained by using formula (22) as

$$
p(x, t)=\sum_{j=1}^{n_{2}} \sum_{j=2}^{n_{1}} p_{i j}
$$

Due to the Bayesian formula

$$
p\left(x, \frac{t_{k+1}}{z_{k+1}}\right)=\frac{p\left(z_{k+1} / x\right) p\left(x, t_{k+1}^{-} / z_{k}\right)}{\iint_{\Omega} p\left(z_{k+1} / x\right) p\left(x, t_{k+1}^{-} / z_{k}\right) d x d y},
$$

the likelihood probability density is defined as

$$
\begin{aligned}
& p\left(\frac{z_{k+1}}{x}\right) \\
& =\frac{\exp \left\{-(1 / 2)\left(z_{k}-h\left(x, t_{k}\right)\right)^{T} R_{k}^{-1}\left(z_{k}-h\left(x, t_{k}\right)\right)\right\}}{\sqrt{(2 \pi)^{m} \operatorname{det} R_{k}}},
\end{aligned}
$$

where $h\left(x, t_{k}\right)$ is a two-dimensional measurement function, $z_{k}$ represents the measurement in a two-dimensional space, and $R_{k}=\left(\begin{array}{cc}\operatorname{Var}\left(x_{1}\right) & \operatorname{Cov}\left(x_{1}, x_{2}\right) \\ \operatorname{Cov}\left(x_{2}, x_{1}\right) & \operatorname{Var}\left(x_{2}\right)\end{array}\right)$ is a covariance matrix of the posterior probability density.

By substituting the prior probability density into Bayesian formula, the posterior probability density can hold as

$$
\begin{aligned}
p_{i j}\left(x, \frac{t_{k+1}}{z_{k+1}}\right) & \\
= & \frac{p\left(z_{k+1} / x\right) p(x, t)}{\iint_{\Omega} p\left(z_{k+1} / x\right) p(x, t) d x d y}, \quad x \in\left[x_{i-1}, x_{i}\right], \\
& y \in\left[y_{j-1}, y_{j}\right]\left(i=1,2, \cdots, n_{1}, j=1,2, \ldots, n_{2}\right),
\end{aligned}
$$

where $p\left(x, t_{k+1} / z_{k+1}\right)$ is a piecewise function as well.

According to the posterior probability density, the conditional mean $\mathrm{E}\left(x / z_{k+1}\right) \triangleq \mathrm{E}_{k+1}$ and the variance $\sigma\left(x / z_{k+1}\right) \triangleq$ $\sigma_{k+1}$ of a system can be obtained by the following equations

$$
\begin{gathered}
\mathrm{E}\left(\frac{x}{z_{k+1}}\right)=\iint_{\Omega} p\left(x, \frac{t_{k+1}}{z_{k+1}}\right) d x_{1} d x_{2} \\
\sigma\left(\frac{x}{z_{k+1}}\right)=\iint_{\Omega}\left(x-\mathrm{E}\left(\frac{x}{z_{k+1}}\right)\right)^{2} p\left(x, \frac{t_{k+1}}{z_{k+1}}\right) d x_{1} d x_{2} .
\end{gathered}
$$

\section{Convergence Analysis}

In this section, we will give the analysis of the proposed estimating method based on FEM. To make the convergence arguments transparent, some special notations are introduced in the process. Assume that $\Omega=\left\{\left(x_{1}, x_{2}\right) \mid a \leq x \leq\right.$ $b, c \leq y \leq d\}$ is a closed and bounded subset of $R^{2}$, and $P(x, t)$ represents any continuous functions in $\Omega$. Suppose that the prior probability density function in each subunit is continuous. For any two adjacent subintervals $\left\{\left(x_{1}, x_{2}\right)\right.$ | $\left.a_{i-1}<x_{1}<a_{i}, b_{i-1}<x_{2}<b_{i}\right\},\left\{\left(x_{1}, x_{2}\right) \mid a_{i}<x_{1}<a_{i+1}, b_{j}<\right.$ $\left.x_{2}<b_{j+1}\right\}$, in the first unit $\left\{\left(x_{1}, x_{2}\right) \mid a_{i-1}<x_{1}<a_{i}, b_{j-1}<\right.$ $\left.x_{2}<b_{j}\right\}$, the left limit of the interpolation point $\left(a_{i}, b_{j}\right)$ will be expressed as

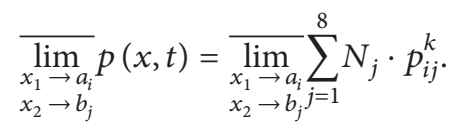

From the shape function described by formula (8), we can get that interpolation point $\left(a_{i}, b_{i}\right)$ corresponds to a shape function $N_{k}$. Obviously, the value of shape function is one, while the remaining values are zero, so

$$
\varlimsup_{\substack{x_{1} \rightarrow a_{i} \\ x_{2} \rightarrow b_{j}}} p(x, t)=N_{k} \varlimsup_{\substack{x_{1} \rightarrow a_{i} \\ x_{2} \rightarrow b_{j}}} p_{i j}^{k}=\varlimsup_{\substack{x_{1} \rightarrow a_{i} \\ x_{2} \rightarrow b_{j}}} p_{i j}^{k} .
$$

By being calculated with (19) and (20), the interpolation point $p_{i j}^{k}$ at $t_{k+1}$ can be obtained as

$$
\begin{aligned}
p^{t_{k+1}}(x) & =p^{t_{k}}(x)+\frac{\Delta t}{6} \cdot\left(K_{1}+2 K_{2}+2 K_{3}+K_{4}\right) \\
& =p^{t_{k}}(x)+F\left(\Delta t+\frac{(\Delta t)^{2}}{3}+\frac{(\Delta t)^{3}}{6}+\frac{(\Delta t)^{4}}{24}\right) .
\end{aligned}
$$

Then,

$$
\begin{aligned}
p^{t_{k+1}}(x)= & p^{t_{k}}(x)+\frac{\Delta t}{6} \cdot\left(K_{1}+2 K_{2}+2 K_{3}+K_{4}\right) \\
= & p^{t_{k}}(x)+F\left(\Delta t+\frac{(\Delta t)^{2}}{3}+\frac{(\Delta t)^{3}}{6}+\frac{(\Delta t)^{4}}{24}\right) \\
= & p^{t_{k}}(x) \\
& +\left(\Delta_{1} p+\Delta_{2} \sum_{i=1}^{2} \frac{\partial p}{\partial x_{i}}+\Delta_{3} \sum_{i, j=1}^{2} \frac{\partial^{2}(p)}{\partial x_{i} \partial x_{j}}\right) \\
& \times\left(\Delta t+\frac{(\Delta t)^{2}}{3}+\frac{(\Delta t)^{3}}{6}+\frac{(\Delta t)^{4}}{24}\right)
\end{aligned}
$$

where $\Delta_{1}, \Delta_{2}, \Delta_{3}$ are only relative to the interpolation point in $F$ and independent with the shape function. Thus, for all $\left(x_{1}, x_{2}\right) \in \Omega$, there exists

$$
\lim _{\substack{x_{1} \rightarrow a_{i} \\ x_{2} \rightarrow b_{j}}} p_{i j}^{k}=p_{i j}^{k}
$$


Therefore,

$$
\varlimsup_{\substack{x_{1} \rightarrow a_{i} \\ x_{2} \rightarrow b_{j}}} p(x, t)=p_{i j}^{k}
$$

In the same way, by choosing the subunit $\left\{\left(x_{1}, x_{2}\right) \mid a_{i-1}<\right.$ $\left.x_{1}<a_{i}, b_{j-1}<x_{2}<b_{j}\right\}$, we can get

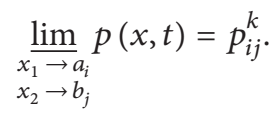

By taking (33) and (34), then

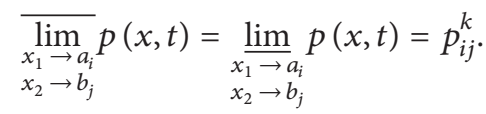

Clearly, $p(x, t)$ is continuous in $\Omega$.

To analyze the convergence of FEM, we shall give the following proof. Suppose that $P(x, t)$ represents all of continuous functions in $\Omega$, for all $p_{1}(x, t), p_{2}(x, t) \in P(x, t)$, and it holds that

$$
d\left(p_{1}, p_{2}\right)=\max _{\substack{a \leq x_{1} \leq b \\ c \leq x_{2} \leq d}}\left|p_{1}(x, t)-p_{2}(x, t)\right| .
$$

Therefore,

(i) for all $p_{1}(x, t), p_{2}(x, t) \in P(x, t), d\left(p_{1}, p_{2}\right)=$ $\max _{a \leq x_{1} \leq b, c \leq x_{2} \leq d}\left|p_{1}(x, t)-p_{2}(x, t)\right| \geq 0$, if and only if $p_{1}=p_{2}, d\left(p_{1}, p_{2}\right)=0$;

(ii) $d\left(p_{1}, p_{2}\right)=\max _{a \leq x_{1} \leq b, c \leq x_{2} \leq d}\left|p_{1}(x, t)-p_{2}(x, t)\right|=$ $\max _{a \leq x_{1} \leq b, c \leq x_{2} \leq d}\left|p_{2}(x, t)-p_{1}(x, t)\right|=d\left(p_{2}, p_{1}\right)$;

(iii) for all $p_{1}(x, t), p_{2}(x, t), p_{3}(x, t) \in P(x, t)$,

$$
\begin{aligned}
d\left(p_{1}, p_{2}\right)= & \max _{\substack{a \leq x_{1} \leq b \\
c \leq x_{2} \leq d}}\left|p_{1}(x, t)-p_{2}(x, t)\right| \\
\leq & \max _{\substack{a \leq x_{1} \leq b \\
c \leq x_{3} \leq d}}\left|p_{1}(x, t)-p_{3}(x, t)\right| \\
& +\max _{\substack{a \leq x_{3} \leq b \\
c \leq x_{2} \leq d}}\left|p_{3}(x, t)-p_{2}(x, t)\right| \\
= & d\left(p_{1}, p_{3}\right)+d\left(p_{3}, p_{2}\right) .
\end{aligned}
$$

So $(P, d)$ is in the metric spaces.

Assume that $p_{n} \in P, n=1,2, \ldots$, is a Cauchy sequence in $P$; for all $\varepsilon>0, \exists N$ is positive integers, and s.t. for all $m, n>$ $N$, there exists

$$
\max _{\substack{a \leq x_{1} \leq b \\ c \leq x_{2} \leq d}}\left|p_{m}(x, t)-p_{n}(x, t)\right|=d\left(p_{m}, p_{n}\right)<\varepsilon .
$$

So for all $\left\{\left(x_{1}, x_{2}\right) \mid a \leq x \leq b, c \leq y \leq d\right\}$, one has $\mid p_{m}(x, t)-$ $p_{n}(x, t) \mid<\varepsilon$, when $t$ is a constant, and $p_{n}(x, t), n=1,2, \ldots$, is a Cauchy sequence. Thus, $\exists p(x, t), p_{n}(x, t) \rightarrow p(x, t), n \rightarrow$ $\infty$. By taking the formula (38), when $n \rightarrow \infty, m>N$, we can get

$$
\max _{\substack{a \leq x_{1} \leq b \\ c \leq x_{2} \leq d}}\left|p_{m}(x, t)-p(x, t)\right| \leq \varepsilon
$$

Above all, for any time $t, p_{m}(x, t)$ is convergent with $p(x, t)$ uniformly for that $p(x, t)$ is continuous. For all $p(x, t) \in P, P$ is completeness, so that $(P, d)$ is a complete function space, and the prior probability density is convergent.

\section{Computational Complexity Analysis}

According to Section 2, we can draw conclusions that for any given area $\Omega \in \mathfrak{R}^{2}$ in a two-dimensional space, the number of the rectangular area can be calculated as $n_{1} \times n_{2}$. Consider that by taking the probability density of subunit area described in (34), it holds that

$$
p_{i j}=\sum_{k=1}^{8} N_{k} \cdot p_{i j}^{k}\left\{(x, y) \mid x_{i-1}<x<x_{i}, y_{j-1}<y<y_{j}\right\}
$$

So the computational complexity of the current interpolation point is

$$
O\left(6 n_{1} \times n_{2}+\left(n_{1}+1\right) \times\left(n_{2}+1\right)+5\left(n_{1}+1\right) \times\left(n_{2}+1\right)\right) .
$$

Thus, the computational complexity of $p_{i j}^{k}$ is $O\left(n_{1} \times\right.$ $\left.n_{2}\right)$. Because the bi-quadratic interpolation method does not involve matrix inversion, finally, in a Bayesian process, the computational complexity of Posterior probability density is $O\left(n_{1} \times n_{2}\right)$ as well. Therefore, the computational complexity of proposed estimating method based on FEM is still $O\left(n_{1} \times n_{2}\right)$.

In addition, we can find that the computational complexity will be growing with a linear speed by the quantity of grid $n_{1} \times n_{2}$. Compared with Particle Filters, assuming that the number of particles is defined as $N$ in a two-dimensional state space and the recursive call was adopted in the process of particle resampling, so the computational complexity is calculated as $O(N !)$. The more number of samples required, the higher computational complexity does PF. Therefore, the computational complexity of the new method based on FEM is simpler than PF.

\section{Illustrative Example and Simulations}

In this section, consider a reference system in a two-dimensional state space given by [27], which is described as follows:

$$
\begin{gathered}
d x=\left[\begin{array}{c}
-x_{2} \\
0.2\left(x_{1}^{2}-1\right) x_{2}+x_{1}
\end{array}\right]+d \beta, \\
z_{t_{k}}=\left(\begin{array}{c}
\sqrt{x_{1}^{2}+x_{2}^{2}} \\
\arctan \left(\frac{x_{1}}{x_{2}}\right)
\end{array}\right)+e_{t_{k}}
\end{gathered}
$$

where the covariance of $d \beta$ is $\operatorname{diag}(10,0.1)$, and the covariance of measurement noise $e_{t_{k}}$ is $\operatorname{diag}(0.1,1)$. For any time $e_{t_{k}}$, it is a two-dimensional white Gaussian sequence independent of $d \beta$. 


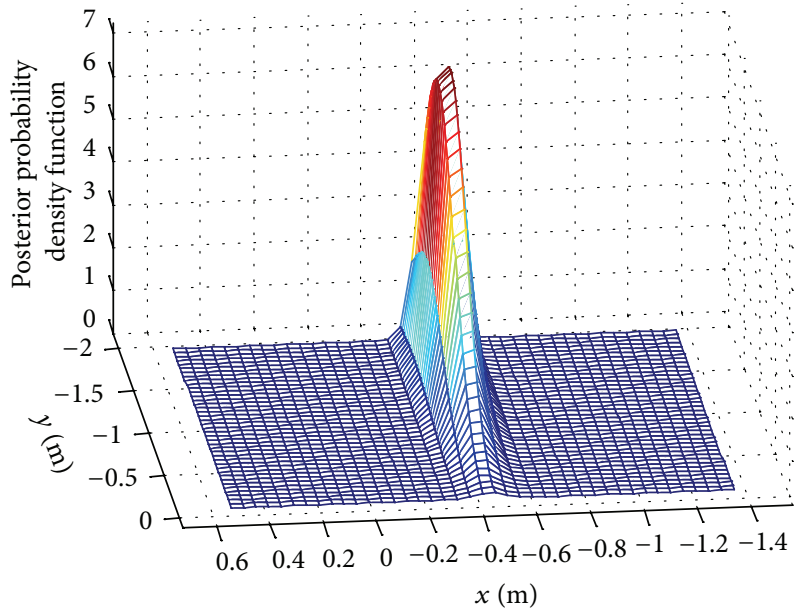

(a)

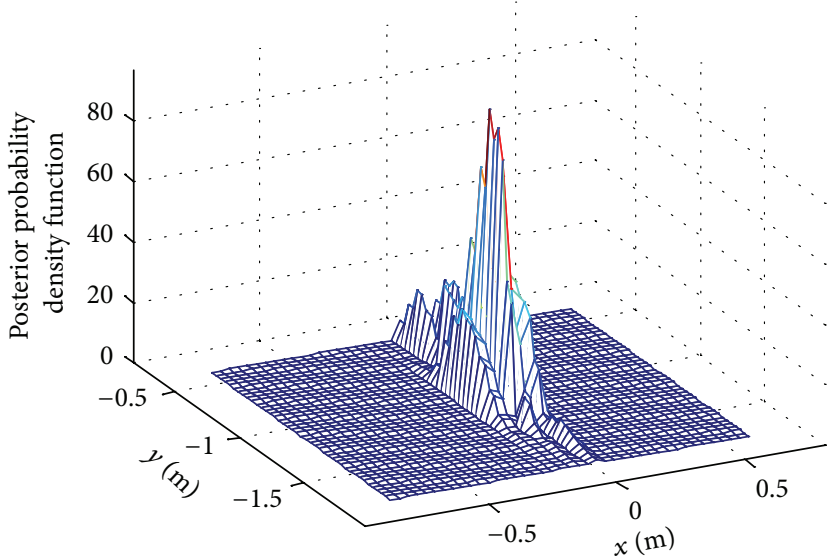

(b)

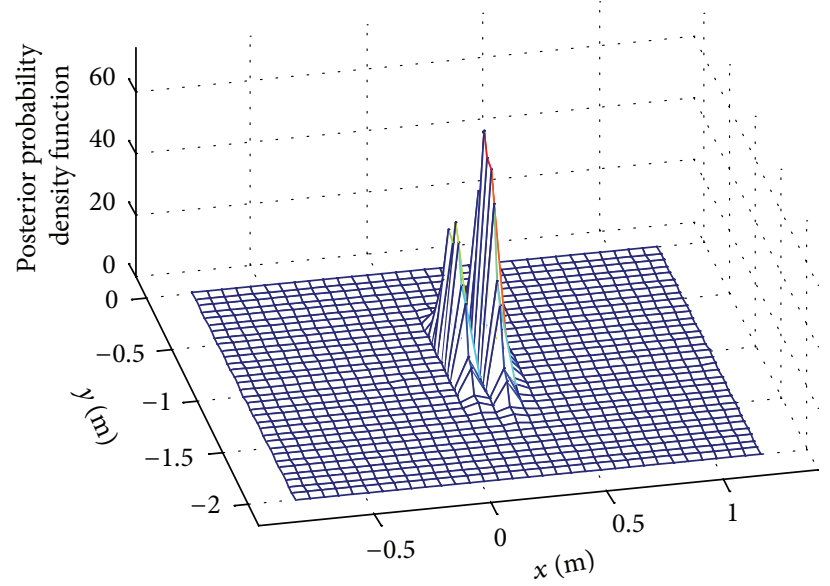

(c)

Figure 3: (a) Approximate density function and the true state at the measurement updates at 1 second. (b) Approximate density function and true state after the measurement updates at 12 seconds. (c) Approximate density function and true state after the measurement updates at 28 seconds.

Here, for FEM, the interval of time is chosen as $1 \mathrm{~s}$, the quantity of grid is chosen as 20 , and the initial state is $[-0.6-1]$. For PF, the number of particles is chosen as 200 .

The performance output of FEM is shown in Figures 3(a), $3(\mathrm{~b})$, and 3(c). The conditional density functions are plotted with discrete times. After a few measurements, the predicted density function is bimodal with modes approximately centered at plus and minus the absolute value of the actual state, which is what we expect. Numerical experiment results show that the feasibility of the proposed stochastic estimating method based on FEM is confirmed.

The true state trajectory and the conditional means of the trajectories executed by FEM, UKF, and PF are given in Figures 4(a) and 4(b). Obviously, for a nonlinear nonGaussian system, UKF estimating output will lose stableness after a few measurement updates. Because the measurement function of UKF should be linearized with the current mean estimate at each measuring step and the second moment of probability distribution of system state also has to be obtained, but the real distribution of probability may not be normal distribution, the actual state is outside of the region where the linearization is valid. The result is that the UKF estimate jumps outside of the region of attraction. Meanwhile, the FEM performs reasonably well by giving the limited information available from the measurements and does not fail because of the nonlinearities in the system and measurement characteristics.

As the real point in these experiments is generated by the random number, every simulation result will be different, therefore; the estimation precision will be measured by using the root mean square error (RMSE) and standard deviation of state, which is defined as follows:

$$
\begin{aligned}
& R=\frac{\sum_{i=1}^{S T} \sqrt{\left(\sum_{k=1}^{t}\left(S_{k}-Y_{k}\right)^{2}\right) / t}}{S T}, \\
& \sigma=\frac{\sum_{k=1}^{S T} \sum_{i=1}^{t} \sqrt{\mathrm{E}\left(Y_{i}-\mathrm{E} Y_{i}\right)^{2}}}{t \cdot S T},
\end{aligned}
$$




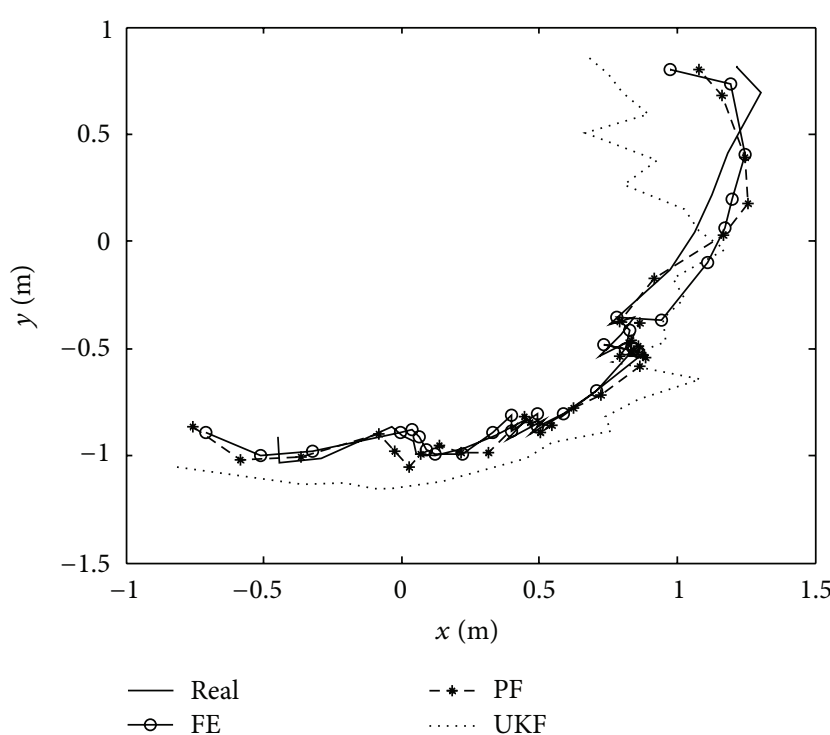

(a)

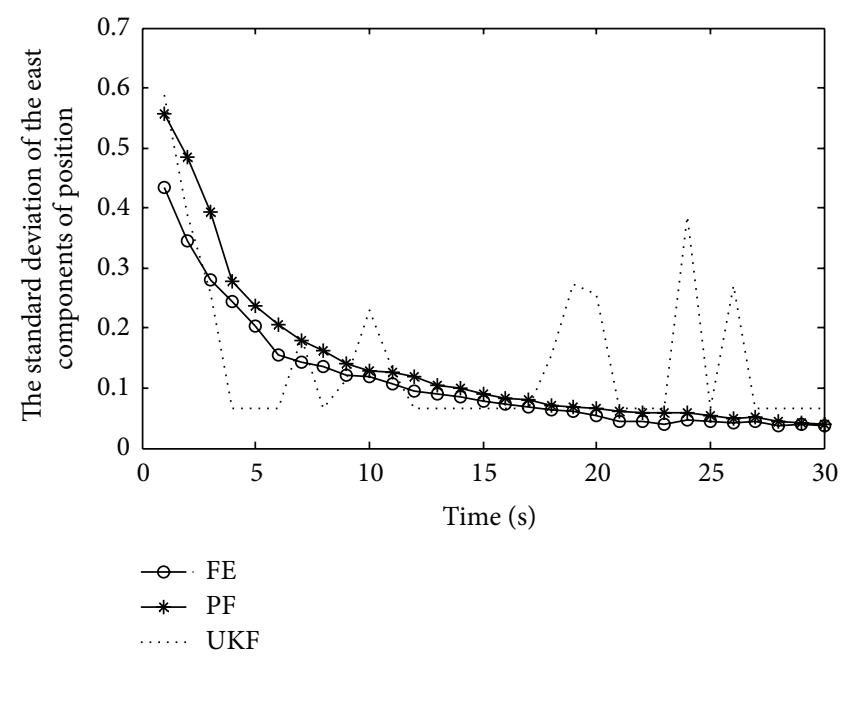

(b)

FIgURE 4: (a) Real state and estimated state trajectories by FEM, PF and UKF. (b) The estimated standard deviation of east components by FEM, UKF, and PF.

TABLE 1: The root-mean-square error and standard deviation of the state.

\begin{tabular}{lcccc}
\hline Algorithm & $R_{x}$ & $R_{y}$ & $\sigma_{x}$ & $\sigma_{y}$ \\
\hline FE & 0.1817 & 0.2250 & 0.0956 & 0.1194 \\
PF & 0.2177 & 0.2444 & 0.1296 & 0.1586 \\
UKF & 0.5366 & 0.4965 & 0.2857 & 0.3223 \\
\hline
\end{tabular}

where $R, \sigma$ are the root mean square error and standard deviation, and $S_{k}, Y_{k}$ are the real position and estimated position. The experiments will be executed 20 times repeatedly.

Clearly, we can see that the performance of FEM is better than PF and UKF shown in Table 1.

\section{Conclusion}

An FEM-based state estimation approach to nonlinear hybrid positioning systems has been investigated in this paper. It was shown that the new filter can be converged to the true density function in a two-dimensional state space. Furthermore, a detailed demonstration for how to use the FEM efficiently is demonstrated, and with a simple example, we can find that FEM outperforms the UKF and the PF reasonably. It is envisioned that the filter could be used to process data from sensors with severely nonlinear output characteristics, and how to implement FEM algorithm in parallel, especially for solving high dimension problem. These considerations should be further extended in our future work.

\section{Acknowledgments}

This work was supported by the National Natural Science Foundation of China (nos. 60904087 and 51109045) and the Fundamental Research Funds for the Central Universities of China (nos. HEUCF110419 and HEUCFX41302).

\section{References}

[1] J. Fujimoto, S. Hotta, K. Sawada, Y. Hada, K. Hida, and S. Mori, "Hybrid positioning system combining spatially continuous and discrete information for indoor location-based service," in Proceedings of the Ubiquitous Positioning, Indoor Navigation, and Location Based Service (UPINLBS '12), pp. 1-6, October 2012.

[2] Y. Gu, A. Lo, and I. Niemegeers, "A survey of indoor positioning systems for wireless personal networks," IEEE Communications Surveys \& Tutorials, vol. 11, no. 1, pp. 13-32, 2009.

[3] M. Ficco and S. Russo, "A hybrid positioning system for technology-independent location-aware computing," Software, vol. 39, no. 13, pp. 1095-1125, 2009.

[4] J. Do, M. Rabinowitz, and P. Enge, "Performance of hybrid positioning system combining GPS and television signals," in Proceedings of IEEE/ION Position Location and Navigation Symposium, pp. 556-564, April 2006.

[5] Y. Lee and M. Kavehrad, "Two hybrid positioning system design techniques with lighting LEDs and ad-hoc wireless network," IEEE Transactions on Consumer Electronics, vol. 58, no. 4, pp. $1176-1184,2012$

[6] P. Kemppi, T. Rautiainen, V. Ranki, F. Belloni, and J. Pajunen, "Hybrid positioning system combining angle-based localization, pedestrian dead reckoning and map filtering," in Proceedings of the International Conference on Indoor Positioning and Indoor Navigation (IPIN '10), pp. 1-7, September 2010.

[7] H. Liu, H. Darabi, P. Banerjee, and J. Liu, "Survey of wireless indoor positioning techniques and systems," IEEE Transactions on Systems, Man and Cybernetics C, vol. 37, no. 6, pp. 1067-1080, 2007. 
[8] S. Ali-Loytty, N. Sirola, and R. Piche, "Consistency of three Kalman filter extensions in hybrid navigation," in Proceedings of the European Navigation Conference (GNSS '05), July 2005.

[9] T. Perälä and R. Piché, "Robust extended Kalman filtering in hybrid positioning applications," in Proceedings of the 4th Workshop on Positioning, Navigation and Communication (WPNC '07), pp. 55-63, March 2007.

[10] J. Cuadrado, D. Dopico, J. A. Perez, and R. Pastorino, "Automotive observers based on multibody models and the extended Kalman filter," Multibody System Dynamics, vol. 27, no. 1, pp. 319, 2012.

[11] W. Mei, G. Shan, and C. Wang, "Practical development of the second-order extended Kalman filter for very long range radar tracking," Signal Processing, vol. 91, no. 5, pp. 1240-1248, 2011.

[12] V. A. Bavdekar, A. P. Deshpande, and S. C. Patwardhan, "Identification of process and measurement noise covariance for state and parameter estimation using extended Kalman filter," Journal of Process Control, vol. 21, no. 4, pp. 585-601, 2011.

[13] Y. Shi, K. Sun, L. Huang, and Y. Li, "Online identification of permanent magnet flux based on extended Kalman filter for IPMSM drive with position sensorless control," IEEE Transactions on Industrial Electronics, vol. 59, no. 11, pp. 4169-4178, 2012.

[14] M. Barut, R. Demir, E. Zerdali, and R. Inan, "Real-time implementation of Bi input-extended Kalman filter-based estimator for speed-sensorless control of induction motors," IEEE Transactions on Industrial Electronics, vol. 59, no. 11, pp. 4197-4206, 2012.

[15] C. Eling, P. Zeimetz, and H. Kuhlmann, "Development of an instantaneous GNSS/MEMS attitude determination system," GPS Solutions, vol. 17, no. 1, pp. 129-138, 2013.

[16] N. J. Gordon, D. J. Salmond, and A. F. M. Smith, "Novel approach to nonlinear/non-gaussian Bayesian state estimation," IEE Proceedings F, vol. 140, no. 2, pp. 107-113, 1993.

[17] M. K. Pitt and N. Shephard, "Filtering via simulation: auxiliary particle filters," Journal of the American Statistical Association, vol. 94, no. 446, pp. 590-599, 1999.

[18] R. van der Merwe, N. de Freitas, A. Doucet, and E. Wan, “The unscented partical filter," in Advances in Neural Information Processing Systems, 2000.

[19] J. H. Kotecha and P. M. Djurić, "Gaussian particle filtering," IEEE Transactions on Signal Processing, vol. 51, no. 10, pp. 25922601, 2003.

[20] A. Doucet and O. de Freitas, "Rao-Blackwellised particle filtering for dynamic bayesian networks," in Proceedings of the 16th Conference on Uncertainty in Artificial Intelligence, pp. 176-183, June 2000.

[21] S. Park, J. P. Hwang, E. Kim, and H.-J. Kang, "A new evolutionary particle filter for the prevention of sample impoverishment," IEEE Transactions on Evolutionary Computation, vol. 13, no. 4, pp. 801-809, 2009.

[22] Y. Zhao and L. Chen, "Explore on cubic spline interpolation for solving navigation stochastic differential model," Control Theory and Applications, vol. 28, no. 8, pp. 987-993, 2011.

[23] I. Kalashnikova, C. Farhat, and R. Tezaur, "A discontinuous enrichment method for the finite element solution of high Péclet advection-diffusion problems," Finite Elements in Analysis and Design, vol. 45, no. 4, pp. 238-250, 2009.

[24] M. A. El-Gebeily and H. E. Emara Shabaik, "Approximate solution of the Fokker-Planck-Kolmogorov equation by finite elements," Communications in Numerical Methods in Engineering, vol. 10, no. 10, pp. 763-771, 1994.
[25] O. Lehtikangas, T. Tarvainen, V. Kolehmainen, A. Pulkkinen, S. R. Arridge, and J. P. Kaipio, "Finite element approximation of the Fokker-Planck equation for diffuse optical tomography," The Journal of Quantitative Spectroscopy \& Radiative Transfer, vol. 111, no. 10, pp. 1406-1417, 2010.

[26] A. Masud and L. A. Bergman, "Application of multi-scale finite element methods to the solution of the Fokker-Planck equation," Computer Methods in Applied Mechanics and Engineering, vol. 194, no. 12-16, pp. 1513-1526, 2005.

[27] R. Beard, J. Gunther, J. Lawton, and W. Stirling, "Nonlinear projection filter based on galerkin approximation," Journal of Guidance, Control and Dynamics, vol. 22, no. 2, pp. 258-266, 1999. 


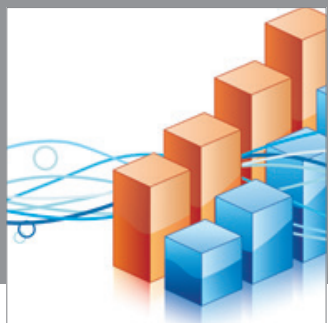

Advances in

Operations Research

mansans

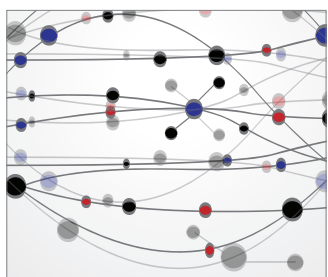

The Scientific World Journal
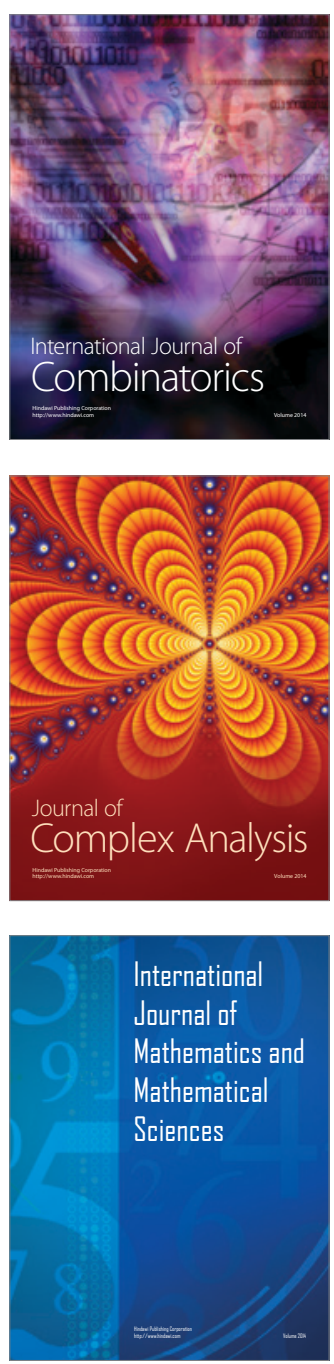
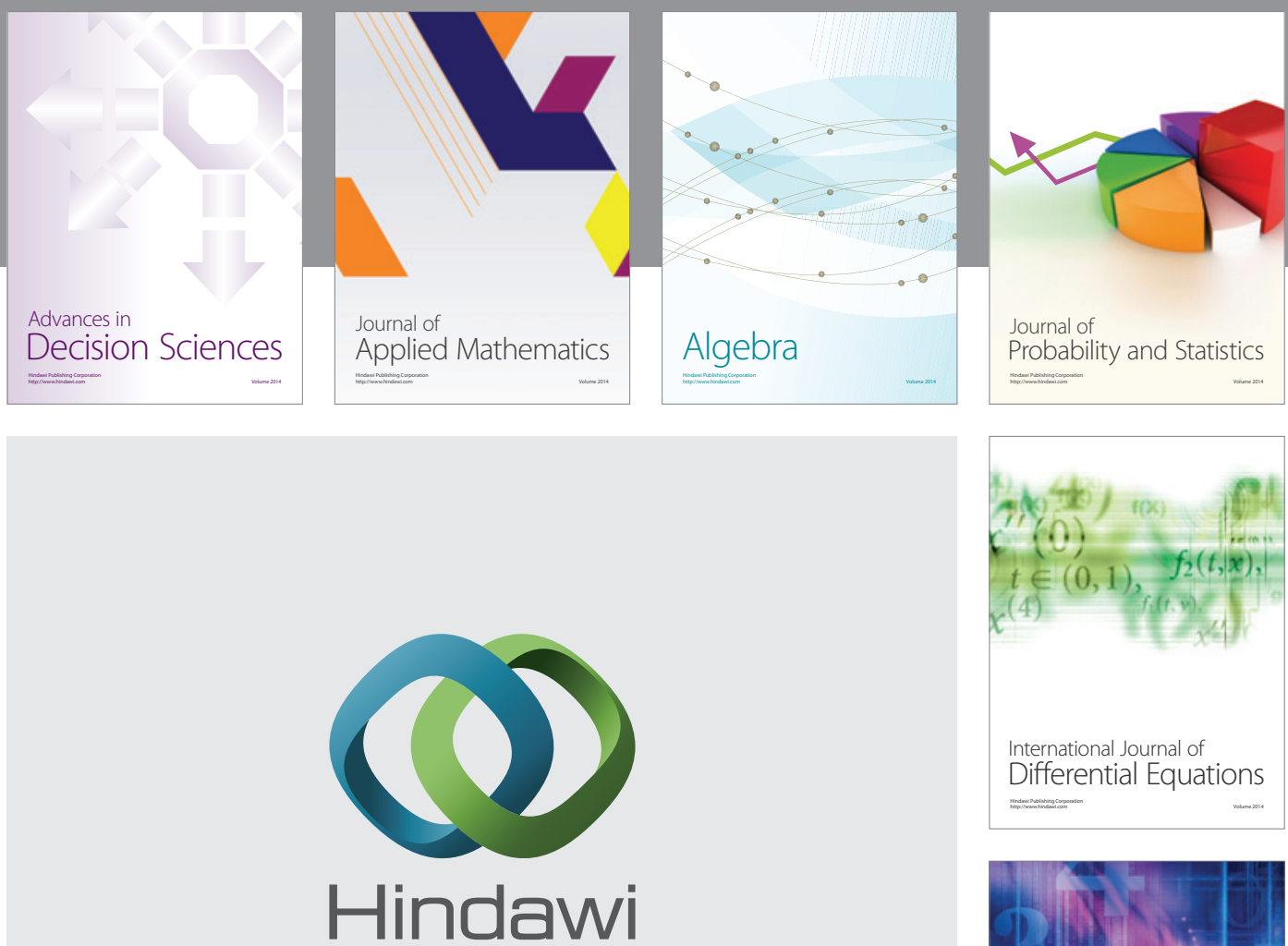

Submit your manuscripts at http://www.hindawi.com
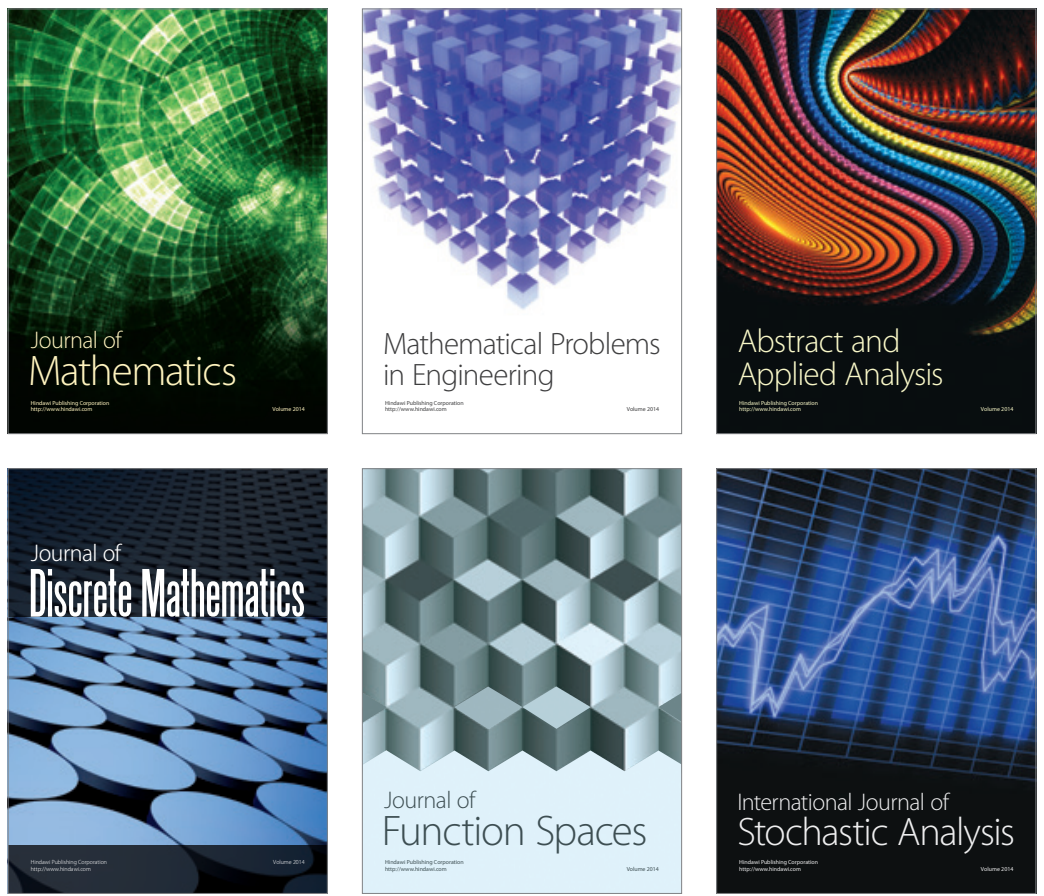

Journal of

Function Spaces

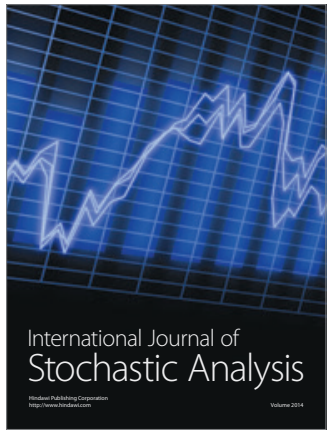

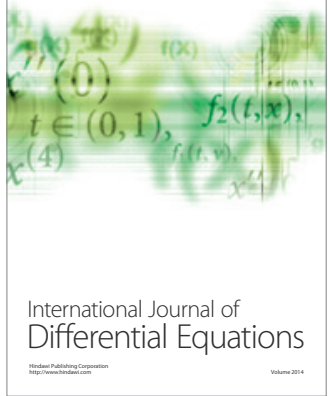
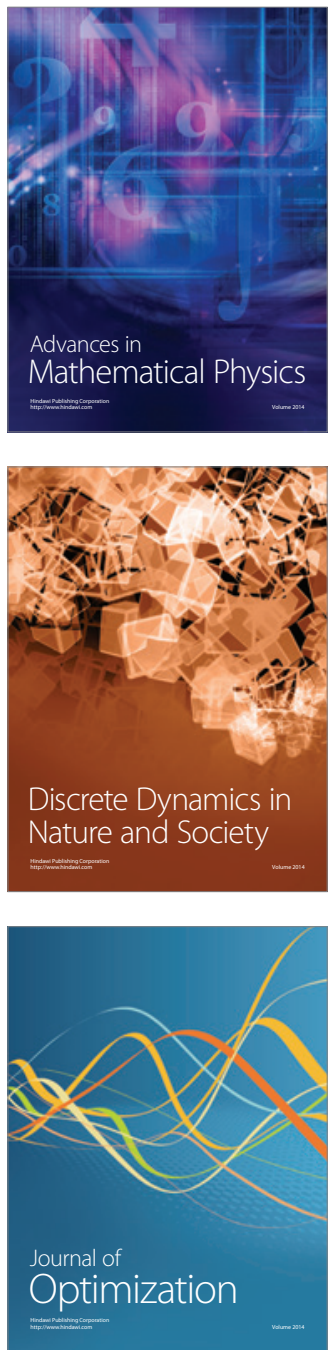